\title{
Using kernels and ecological niche modeling to delineate conservation areas in an endangered patch-breeding phenotype
}

\author{
Mathieu DenoëL ${ }^{1,4}$ and Gentile Francesco Ficetola ${ }^{2,3}$ \\ ${ }^{1}$ Laboratory of Fish and Amphibian Ethology, Behavioural Biology Unit, University of Liege, 22 Quai van Beneden, 4020 Liege, Belgium \\ ${ }^{2}$ Department of Environmental and Earth Sciences, University of Milano-Bicocca. Piazza della Scienza 1, 20126 Milano, Italy \\ ${ }^{3}$ Laboratory of Alpine Ecology (LECA), UMR CNRS 5553, Joseph Fourier University, 38041 Grenoble, France
}

Abstract. Efficient delineation of conservation areas is a great challenge in maintaining biodiversity. Kernel density estimators (KDEs) are a powerful tool in this perspective, but they have not been applied at the population level on patch-distributed organisms. This would be particularly worthy for species that need broad habitats beyond those where they can be sampled; such as terrestrial lands for pond-breeding amphibians. The aim of this study was to compare different approaches for the identification of suitable areas for conservation: KDE, ecological niche modelling, and a combination of KDE and niche models. Paedomorphosis was chosen as a model system because this is an important form of intraspecific variation that is present in numerous taxa, but geographically localized within species and globally endangered. 277 ponds were sampled in one of the hotspots of paedomorphosis to determine the abundance and distribution of paedomorphs (i.e., individuals retaining gills at the adult stage) of the palmate newt (Lissotriton helveticus), with emphasis on the connections between the most valuable populations. KDEs gave insights into the surface areas required to balance the maintenance of certain number of connected ponds and the respective number of disjoint areas in which the whole population is divided. The inclusion of barriers in the models helped in accurately designing the limits of the areas to protect. Alone, habitat models were not able to successfully delineate the area to protect, but the integration between terrestrial suitable areas or barriers and KDE allowed an objective identification of areas required for conservation. Overall, the best performance was observed by the KDE integrating ecological barriers, and by the combination between KDE and niche modelling. In a broader perspective, KDEs are thus a pertinent tool in providing quantitative spatial measurements to delineate conservation areas based on patch-abundance data with a specific focus to connectivity.

Key words: amphibian decline; connectivity; conservation; ecological niche model; facultative paedomorphosis; kernel density estimation; MaxEnt; palmate newt; protected areas; utilization distribution.

\section{INTRODUCTION}

Identifying and delineating conservation areas are of primary importance to sustain biodiversity. This has proven useful at large scales by focusing priorities on global biodiversity hotpots (Myers et al. 2000). At more local scales, this approach allows the characterization of more strictly protected areas such as reserves or national parks based on the distribution or densities of threatened species and communities (Ronconi et al. 2012). In this framework, taking into account connectivity and broad scale area is also an essential strategy in designing conservation areas (Cushman et al. 2012, Kanagaraj et al. 2013). Historically, the identification of conservation areas has been based on subjective criteria. However, in the present biodiversity crisis, quantitative approaches are needed for an effective identification of areas to be

Manuscript received 2 June 2014; revised 12 November 2014; accepted 28 January 2015. Corresponding Editor: T. W. J. Garner.

${ }^{4}$ E-mail: Mathieu.Denoel@ulg.ac.be prioritized for conservation (Kremen et al. 2008, Naidoo et al. 2008).

The development of spatial statistical methods now offers the possibility of more accurately defining conservation boundary limits. Several tools, such as ecological niche modelling (ENM; following Warren [2012]), landscape genetics (Segelbacher et al. 2010), and kernel density estimations (KDEs; O'Brien et al. 2012) have been proposed to identify suitable areas for target species, and provide objective indication where to put the boundaries for protected areas. Landscape genetics is mostly used to evaluate connections between populations and to propose corridors (Segelbacher et al. 2010). ENMs can evaluate relationships between species distribution data and environmental layers representing both landscape and climatic variables, and identify areas with highest environmental suitability. These suitability maps can be then used to propose areas with high conservation priority, particularly for threatened species for which limited information on distribution is available (Araujo and Williams 2000, Pearson 2011). Kernels have initially been widely used to delineate home ranges, 
i.e., the amount of space used by animal individuals (Worton 1989, Hart et al. 2013). They consist of estimating density probabilities across space (Seaman and Powell 1996). Such location data are obtained from visual or automated tracking of individuals, but given their costs are often limited to a fraction of the population (Wilson et al. 2009, Hart et al. 2013, Schofield et al. 2013). KDEs can also take into account barriers to better delineate distributions utilization (Benhamou and Cornélis 2010, Barry and McIntyre 2011), but such elements are rarely taken into account.

More recently, KDEs have been proposed to characterize the distribution and abundance of species across space (Martins et al. 2013). In this context, the location of each observation of different individuals at a given time is used instead of the location of a single individual across time. The analyses thus allow one to explore areas larger than just the home ranges and to take the whole population into consideration, and thus to delineate conservation areas that encompass the highest number of individuals. For instance, O'Brien et al. (2012) used bird surveys along transects to define the limits of a coastal reserve. However, such analyses have not yet been done on the basis of patch-breeding occupancy data. Such an approach would be particularly useful for species that can be sampled efficiently in small patches, whereas they need surrounding environments where they have more secretive habits during their life cycle. Amphibian decline is the highest among vertebrates, and the amphibian biodiversity crisis has been described as the sixth world mass extinction (Wake and Vredenburg 2008). Pond-breeding amphibians need water for reproduction but also terrestrial lands for foraging, estivation and overwintering (Wells 2007). Their biphasic life cycle makes them particularly vulnerable to environmental changes as disturbance in both aquatic and terrestrial lands could have detrimental consequences. In addition of providing adequate resources, the terrestrial environment is essential in maintaining connectivity among breeding sites (Smith and Green 2005). On the other hand, although some species are abundant and widespread over large areas, others are locally rare over their distribution range or are geographically localized (Sillero et al. 2014). It is thus essential to delineate connected conservation areas that encompass both aquatic and terrestrial habitats, rather than focusing only on the conservation of wetlands. In this context, KDEs could prove useful in determining distribution utilization areas, but the method remains to be applied on an empirical case.

Some species exhibit phenotypic variation in having alternative morphs that differ in their distribution ranges (Tarjuelo et al. 2004, Denoël 2007). This is the case of facultative paedomorphosis in newts and salamanders, a process in which some individuals mature while retaining gills (the paedomorphs), whereas the others metamorphose into a terrestrial morph (the metamorphs) that come back to water to breed (White- man 1994). Populations with paedomorphic individuals are rare, threatened and geographically clustered. They constitute evolutionarily significant units that are essential to maintaining intraspecific diversity (Crandall et al. 2000, Denoël 2007). In Europe, two main areas have been associated with the highest prevalence of paedomorphosis: Larzac in France and Montenegro (Denoël 2007, Denoël et al. 2009). Facultative paedomorphosis has been shown to have a genetic component (Johnson and Voss 2013), but this is also an environmentally cued polymorphism (Semlitsch 1987). Since paedomorphic populations are particularly threatened at the global scale, there is a need for active protection (Denoël et al. 2005). However, there are no quantitative guidelines indicating which specific areas should be protected for maintaining these populations.

The aim of this study was to evaluate whether KDEs, ENM, or a combination of these two approaches can provide quantitative guidelines for mapping protection areas of patch-breeding species. As variables such as barriers and land cover could influence utilization distributions (Benhamou and Cornélis 2010), we evaluated whether integrating these elements into KDE analyses can improve the outcome of analyses. To this end, we used populations of paedomorphic palmate newts (Lissotriton helveticus) in Larzac as a model system for four main reasons: (1) the study area is inhabited by a high rate of populations with paedomorphosis, allowing quantitative analyses $(68 \%$ of the known cases of paedomorphosis in the palmate newt have been identified in this area, which comprises $0.5 \%$ only of the species distribution range); (2) paedomorphosis is not uniformly distributed across space; (3) all paedomorphs are limited to distinctly individualized patches, i.e., ponds; (4) paedomorphs are locally and globally declining because of environmental modifications, thus making it essential to delineate areas where conservation efforts are explicitly targeted towards them (Denoël et al. 2005, Denoël 2007, Denoël and Ficetola 2014).

\section{MATERIALS AND METHODS \\ Pond locations and newt sampling}

We determined the distribution and local abundance of paedomorphic palmate newts by surveying 277 ponds in southern Larzac (France). The area studied, delineated by a minimum convex polygon (MCP) computed on the full set of ponds, covered $442 \mathrm{~km}^{2}$. Larzac is a rural area with low urbanization and a high abundance and diversity of amphibians (Gabrion et al. 1977, Denoël and Lehmann 2006, Geniez and Cheylan 2012).

Ponds were sampled once a year, with an average of 2.4 times per pond during the 12-year study period (2002-2013; i.e., 670 censuses), to obtain an index of paedomorphic newt abundance based on average values across time. Averaged values of paedomorph abundance was chosen because it was not possible to deal with repeated measurements in kernels and to have an 


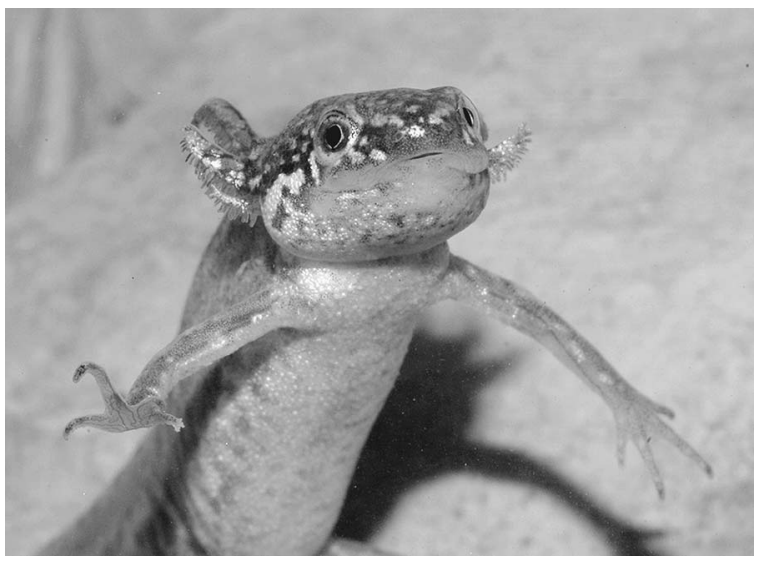

Fig. 1. Paedomorphic palmate newt (Lissotriton helveticus).

average representation of the interest of each pond. Paedomorphs differ from metamorphs on multiple traits, such as the presence of gills only present in the paedomorphs (Fig. 1). Their adulthood was established by the presence of a developed cloaca. Newts were caught by dip-netting and seining along transects to cover the entire surface of the ponds several times, including the deepest parts (Denoël and Lehmann 2006). Sampling ended after many unsuccessful netting attempts in varied areas of the ponds. This method has the advantage of sampling all micro-habitats and thus gives comparable values across ponds since all ponds were surveyed similarly by the same person. Previous studies showed that the individual detection probability with this type of removal estimate is very high (Wilbur 1997) and that using dip nets is an adequate method for sampling newts (Arntzen 2002).

\section{Statistical analyses}

Kernel density estimation.-Fixed normal kernel density estimations were produced on the basis of the distribution of paedomorphic individuals, by using the index of abundance at each pond for the calculation. For kernel analysis, we used a cell size of $0.01 \mathrm{~km}^{2}$. We used the ad hoc method for the optimum choice of the smoothing parameter of the kernel $h$, which controls the "width" of kernel functions placed over each point (Worton 1989, 1995): $h=\mathrm{s} \times n^{-1 / 6}$ where $n$ is the sample size, $s=\left(0.5 \times\left[s_{x}^{2}+s_{y}^{2}\right]\right)^{1 / 2}$, and $s_{x}^{2}, s_{y}^{2}$ are the estimated variances of $(x, y)$ data. We used a fixed $h$ over the entire surface area, as computer simulations showed that this approach fits data the most accurately (Seaman and Powell 1996). The obtained $h(1.59 \mathrm{~km})$ was coherent with the effect of pond density on newt abundance. Indeed, a previous study highlighted effect of newt abundance at ponds at a distance of $1.3 \mathrm{~km}$ from core ponds (Denoël and Lehmann 2006). We considered 10 KDE values from $10 \%$ to $95 \%$ (Fig. 2A); each KDE value is delineated by its respective isopleth (i.e., line on a map) and corresponds to the smallest area protecting, respec- tively, $10-95 \%$ of individuals. In our analyses, we were interested in both coverages and number of disjoint areas obtained using the different KDE values. Both parameters are important for conservation planning, as for amphibians, broad areas are expected to protect more connected ponds whereas isolated and disjoint areas often have lower conservation effectiveness than the connected ones (Marsh and Trenham 2001).

Southern Larzac is a plateau that is naturally limited by a mountain ridge to the south. A canyon separates the two plateaus (Larzac sensu stricto and Blandas) studied in Larzac. The Blandas plateau is limited northward by the Arre Valley. In addition, a highway limits the study area on its western edge (Fig. 2B). These natural and anthropogenic features could constitute strong or even impassable barriers for newt movement. Therefore, kernel analyses were repeated while taking into account the effect of barriers (Benhamou and Cornélis 2010). Barriers were drawn following the limits of the plateau and the main highway using GIS data (digital elevation model and road data from the Institut Géographique National, Saint-Mandé, France). During amphibian surveys, detection probability is generally less than $100 \%$ (Mazerolle et al. 2007). Unfortunately, detection probability cannot be integrated into kernel analysis. Nevertheless, previous analyses showed that detection probability with the survey methods is very high in the study area, therefore, analyses not taking into account detection probability yield results that are nearly identical to analyses integrating detection probability (Denoël and Ficetola 2014). In order to depict the effect of the two kernel approaches (without/with barrier) and of the KDE values (i.e., isopleths of different percentages, from $10 \%$ to $95 \%$ ) on the surface of the utilization distribution, we used linear mixed models. Area was used as dependent variable while approach (Kernel without and with barriers), KDE value, and their interaction were considered as independent variables. Areas at the same KDE are not independent because they are multiple measurements taken on the same data, therefore the identity of KDE values was considered a random effect.

Ecological niche modelling.-We used Maximum Entropy Modeling (MaxEnt) to build ENMs relating the distribution of paedomorphs with environmental variables. MaxEnt is a presence-background approach that evaluates the suitability of a given cell on the basis of environmental features in that cell (Phillips et al. 2006, Elith et al. 2011). Comparative analyses showed that MaxEnt is among the ENMs with best predictive performance (Elith et al. 2006, 2011). Two topographic features were used from the digital elevation model: elevation above sea level and slope in a $100-\mathrm{m}$ resolution raster. As a measure of landscape composition (availability of terrestrial habitat), we used the percentage covered by natural vegetation in the $100-\mathrm{m}$ cell, calculated on the basis of CORINE Landcover 2006. As presence points, we used the location of ponds 
A

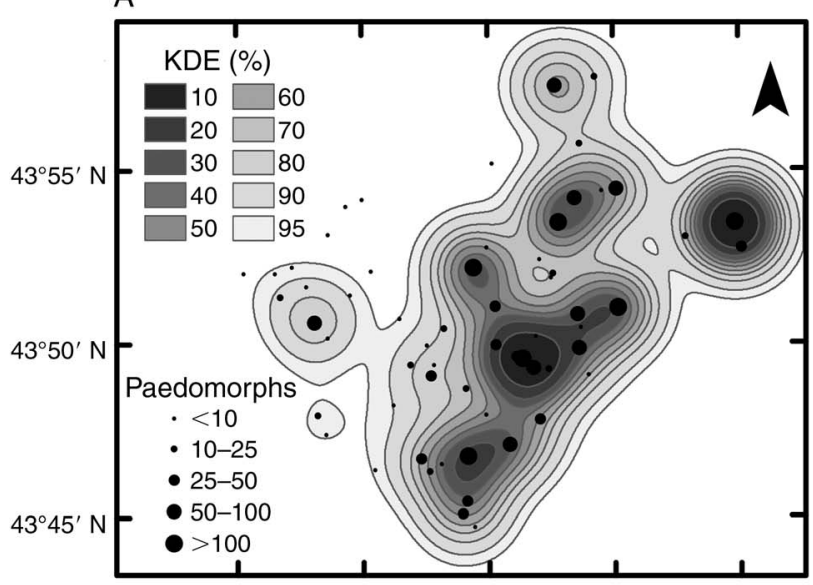

C

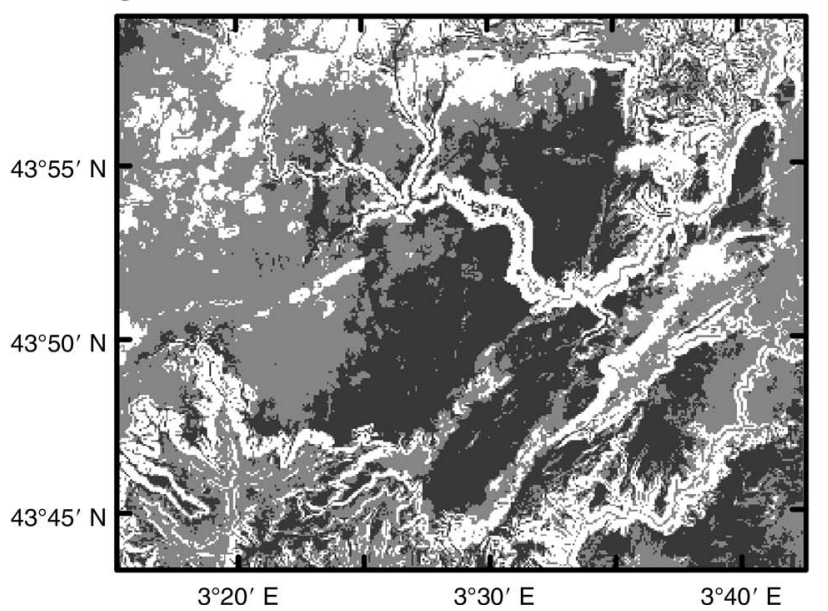

B

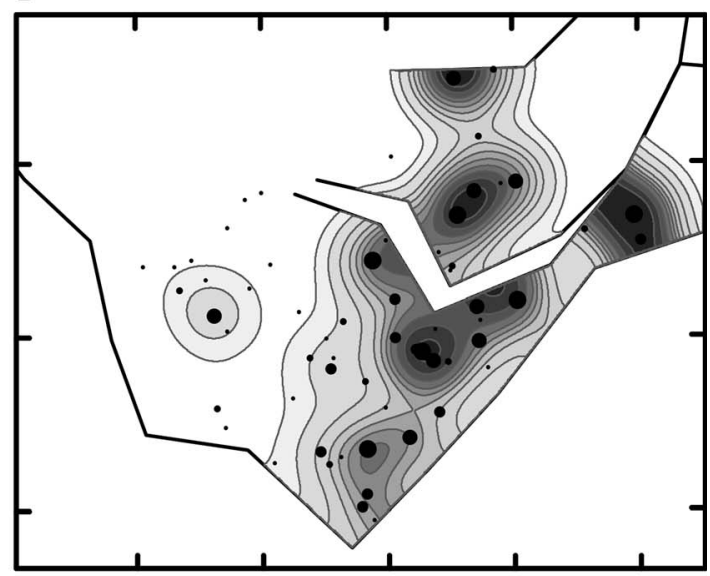

D

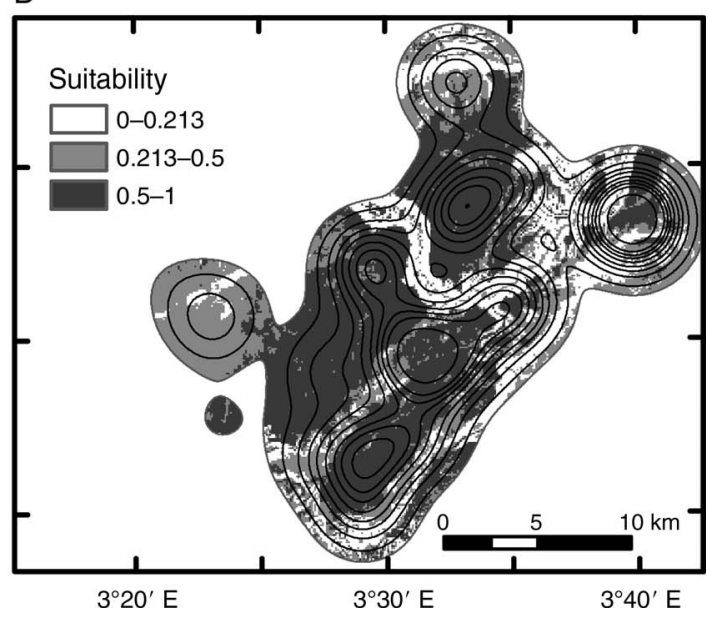

FIG. 2. Cartographic representation of kernel density estimators (KDEs) and ecological niche model (MaxEnt) for paedomorphic palmate newts (Lissotriton helveticus) in southern Larzac (France): (A) KDE without barriers; (B) KDE with barriers; (C) MaxEnt; (D) MaxEnt combined with KDE. The average number of paedomorphs caught per pond is proportional to the size of full circles $(n=61$ ponds). KDEs from $10 \%$ to $95 \%$ are shown. For MaxEnt models, 0.213 is the minimum training presence suitability threshold. In panel (B), the barriers separate two plateaus: the Larzac plateau in the south and west and the Blandas plateau in the north and east. Barriers correspond to both anthropogenic features (highway) and natural features (limits of the plateau by canyons and a deep valley).

containing the main populations of paedomorphic palmate newts ( $n>10$ paedomorphs). This approach was used as it gives greater emphasis to ponds with many paedomorphs (as in KDE). MaxEnt models calibrated using all the ponds with paedomorphs instead than using that only those with more than 10 paedomorphs yielded identical results (correlation between the two ouputs: $r=0.95$ ). We performed censuses in all ponds for the area within the MCP of 277 ponds, while areas outside the MCP were only occasionally surveyed. Therefore, we assumed a sampling bias within the MCP, with a 10 times better sampling within this area (Phillips et al. 2009). This bias value was chosen following recommendations of Kramer-Schadt et al. (2013). Preliminary analyses using different values of bias yielded identical results. We used a 10 -fold crossvalidation to evaluate the performance of the model.
Data were split into 10 sets. We built a model using $90 \%$ of the data (calibration data) and tested predictive performance for the remaining $10 \%$ of the data (test data). This procedure was repeated 10 times, each time using a different set of test data (Nogués-Bravo 2009). As a measure of model performance, we calculated the area under the curve (AUC) of the receiver operator plot for the test data and averaged over the ten replicated runs as a measure of predictive performance (Manel et al. 2001). We assumed that a cell is suitable if it shows suitability higher than the minimum training presence threshold (averaged over the 10 cross-validated runs), while we assumed a high suitability if suitability is higher than 0.5 (Elith et al. 2011).

In preliminary analyses, we also tested a model including additional variables representing landscape composition and human land-cover (altitude, slope, 
cover of open pastures and cropland, forest, scrubland, sclerophyllous vegetation, and urban areas). However, at landscape scale MaxEnt models often show better performance when only a small number of variables is used (Ficetola et al. 2014). In our study, the model with seven variables showed a much higher Akaike information criterion corrected for small sample size $\left(\mathrm{AIC}_{\mathrm{c}}=\right.$ $990.0)$ than the model with just three variables $\left(\mathrm{AIC}_{\mathrm{c}}=\right.$ 970.4). Therefore, we present the results of the threevariable model, which pooled the main variables representing natural habitat (see Warren and Seifert 2011).

Comparison of performance across approaches.-We used a cross-validation approach to assess the performance of the four approaches (KDE without barriers, KDE with barriers, Maxent and a combination of KDE with MaxEnt). The data set of ponds with paedomorphs was divided in 10 groups. Each time, data from $90 \%$ of ponds were used to develop KDE and ENMs, while the remaining $10 \%$ of data was used to test performance (10 runs of cross-validation). For each cross-validation run, the areas that would be protected were delineated using the $90 \% \mathrm{KDE}$ and, for MaxEnt models, by the suitability threshold that would consider $90 \%$ of presence points as being within suitable habitats (i.e., the $10 \%$ training presence threshold; Pearson et al. 2007). Model performance was defined as the ratio between the proportion of paedomorphs that would be protected by a given approach and the proportion of the study area requiring protection. The performance metric therefore favored approaches requiring less area to protect more individuals, as conservation actions are always limited by resources (i.e., large reserves require more resources and are more difficult to establish).

Statistical packages.- The analyses were performed in MaxEnt 3.3.3k (Phillips et al. 2006) and in R 3.0.2 (R Core Development Team 2013), using the packages adehabitatHR (Calenge 2011), dismo (Hijmans et al. 2013), raster (Hijmans 2013), and nlme (Pinheiro et al. 2014). All maps were created with ArcGIS 10.1 (ESRI, Redlands, California, USA).

\section{Results}

During the 12-year study, 8451 paedomorphs were counted over 61 ponds: 50 ponds on the Larzac plateau and 11 on the Blandas plateau (Fig. 2). This partially follows the administrative units as 49 ponds were in the Hérault department and 12 in the Gard department. On average, there were 33.4 paedomorphs per pond and visit $(\mathrm{SE}=6.2$, range over averaged values across years: $0.2-275, n=61$; Fig. 2).

\section{Kernel analysis without barriers}

The $100 \%$ and $95 \%$ MCP for ponds with paedomorphosis covered $350 \mathrm{~km}^{2}$ and $295 \mathrm{~km}^{2}$, respectively, i.e., $79 \%$ and $67 \%$ of the study area. The use of KDEs reduced this area, except at $90 \%$ and $95 \%$ KDE. At $95 \%$, KDE covered an area of $375 \mathrm{~km}^{2}$, whereas it covered 13 $\mathrm{km}^{2}$ at $10 \%$. The surface area defined by each isopleth increased with the KDE values from $10 \%$ to $95 \%$ following a nonlinear pattern (Fig. 3A).

The correlation between number of disjoint areas and $\mathrm{KDE}$ values appeared low (Spearman correlation, $r_{\mathrm{S}}=$ $-0.295, n=10, P=0.41$; Fig. 3B), and only above $90 \%$ $\mathrm{KDE}$ the different delineated areas become more connected (i.e., two instead of three disjoint areas) (Fig. 3B). The number of ponds included in the surface areas delineated by each KDE increased linearly with KDE values, from $10 \%$ to $95 \%\left(r_{\mathrm{S}}=1, n=10, P<0.001\right.$; Fig. $\left.3 \mathrm{C}\right)$. The $50 \%$ KDE area included $39 \%$ of the ponds. The average number of ponds per disjoint areas quickly increased with KDE values $\left(r_{\mathrm{S}}=0.976, n=10, P<0.001\right.$; Fig. $\left.3 \mathrm{D}\right)$.

\section{Kernel analysis with barriers}

The use of barriers modified the utilization distribution (Figs. 2 and 3). The model with barriers generally identified smaller areas than the model without barriers for the different KDE values (e.g., 225 instead of $301 \mathrm{~km}^{2}$ for the $90 \% \mathrm{KDE}$; effect of kernel approach, mixed model, $\left.F_{1,8}=90.7, P<0.001\right)$. However, differences were very limited for the small KDEs (e.g., 12.2 instead of $12.6 \mathrm{~km}^{2}$ for the 10\% KDE; Fig. 3A). Utilization distribution increased as a function of KDEs $\left(F_{1,8}=52.7, P<0.001\right)$, but without barriers the increase was faster than with barriers (interaction between kernel approach and KDE: $\left.F_{1,8}=42.9, P<0.001\right)$.

In contrast to the results obtained with the analysis without using barriers, the number of disjoint areas decreased as KDE values increased $\left(r_{\mathrm{S}}=0.921, n=10\right.$, $P<0.001$; Fig. 3B). The number of disjoint areas was generally higher in the analysis with barriers, if compared to the analysis without barriers (Fig. 3B). The total number of ponds and the mean number of ponds per disjoint area increased with KDEs in a very similar way to the analysis without barriers (Fig. 3C, D). The $50 \%$ KDE area also included $39 \%$ of the ponds. For the analysis on the average number of ponds by disjoint area, a similar value was observed for a $60 \% \mathrm{KDE}$, whereas the analysis with barriers provided a lower number of ponds for all other KDEs (Fig. 3D).

\section{Combining habitat suitability models and Kernel analysis}

Slope and elevation showed the stronger contribution to the model (contribution, $58.9 \%$ and $33.3 \%$, respectively), whereas natural vegetation had a weak effect (7.8\%). Suitability was highest in cells with slope $<10^{\circ}$, altitude of 300-700 m, and with relatively high cover of natural vegetation (Appendix). The model showed generally good predictive performance: the average AUC of cross-validated runs was $0.793(\mathrm{SD}=0.120)$. The minimum training suitability threshold was 0.213 , i.e., all populations showed suitability $\geq 0.213$. A large proportion of the study area $(70 \%)$ showed suitability values higher than this value (Fig. 2C). The model identified potentially highly suitable habitats (i.e., suitability $>0.50$ ) in places where paedomorphs were 

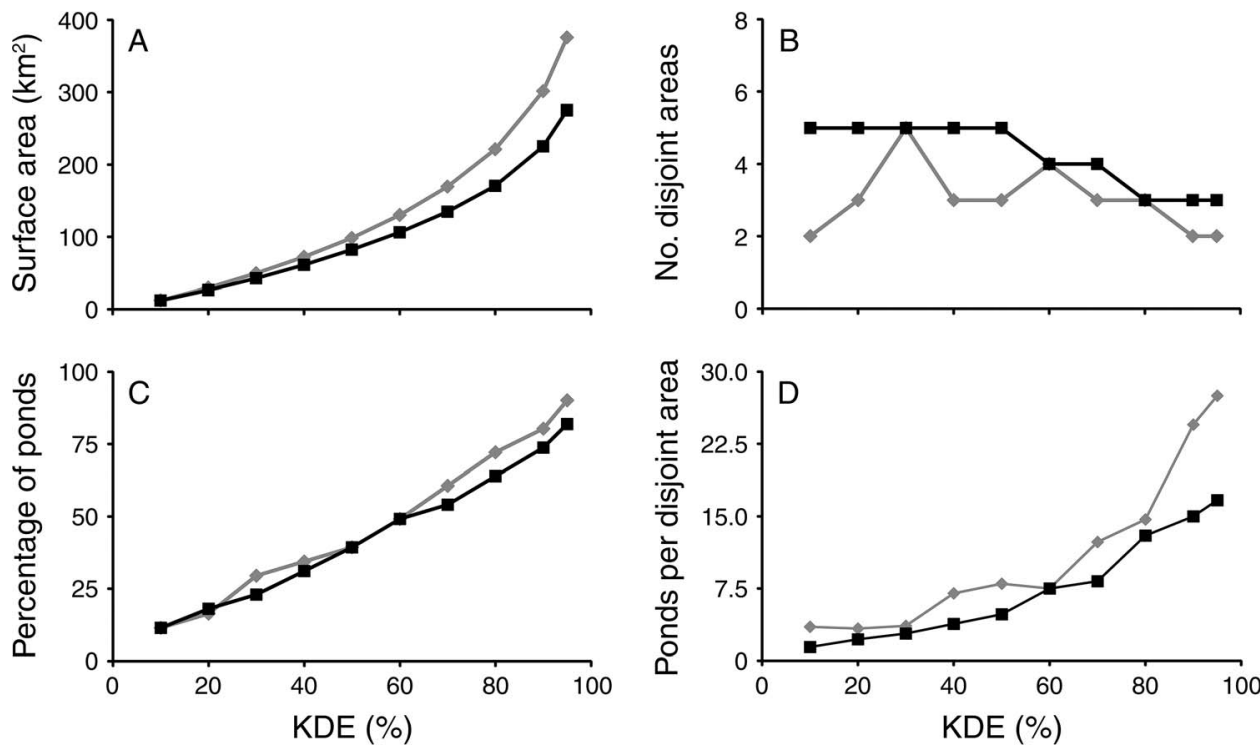

FIG. 3. (A) Surface areas, (B) number of disjoint areas, (C) proportion of ponds with paedomorphs, and (D) mean number of ponds with paedomorphs by disjoint area as a function of kernel density estimators (KDEs) for paedomorphic palmate newts (Lissotriton helveticus) in southern Larzac (France). Gray lines and diamonds show analysis without barriers; black lines and squares show analysis with barriers.

not found, particularly in the southeast of the study area, outside Larzac (Fig. 2C). Combining MaxEnt with KDE highlighted core areas within the limits of each KDE (Table 1). Some areas were considered as unsuitable for newts within each KDE; particularly in the largest KDEs (Table 1).

\section{Comparison of the performance of the four approaches}

Overall, MaxEnt combined with KDE and KDE with barrier were the approaches with the highest performance, while MaxEnt showed the lowest performance (Fig. 4). Across the 10 replicates, MaxEnt combined with KDE consistently showed higher performance than both the

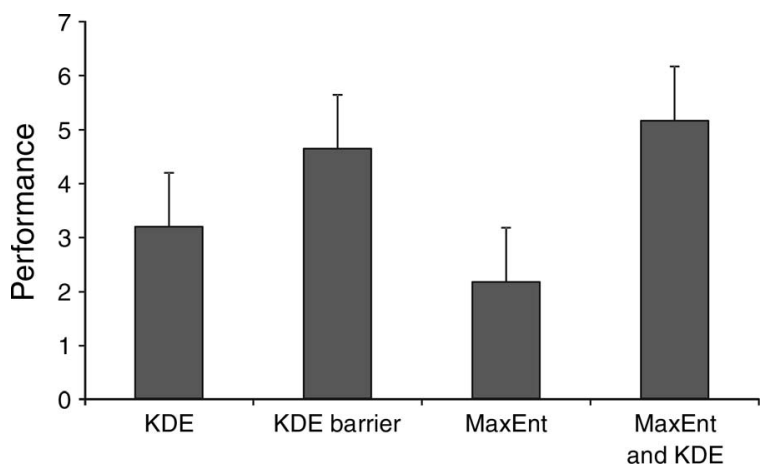

FIG. 4. Performance of the four methods (KDE without barriers, KDE with barriers, MaxEnt, and MaxEnt combined with KDE), evaluated on the basis of cross validation. Performance was evaluated as the ratio between the proportion of paedomorphs that would be protected by a given approach and the proportion of the study area requiring protection. Values are mean $+\mathrm{SD}$.
MaxEnt ( $t$ test for paired samples: $t_{18}=6.0, P<0.001$ ) and the standard KDE approaches $\left(t_{18}=3.3, P=0.005\right)$. The performance of the KDE with barrier and of MaxEnt combined with KDE was very similar $\left(t_{18}=0.7, P=0.49\right)$.

\section{DisCUSSION}

In contrast to analyses not based on distribution probability functions, the use of kernel density estimators allowed us to objectively target the most important areas, i.e., those including connections between the largest populations of paedomorphic individuals. Recent studies highlighted the value of KDEs on the basis of occupancy data, for example along transects (O'Brien et al. 2012). Here, we showed that the method can also

TABLE 1. Surface areas $\left(\mathrm{km}^{2}\right)$ obtained by ecological niche modeling (MaxEnt) within the 10 areas delineated by kernel density estimations (KDE) for paedomorphic palmate newts (Lissotriton helveticus) in Southern Larzac (France) for three different suitabilities.

\begin{tabular}{cccc}
\hline \hline & \multicolumn{3}{c}{ Suitability } \\
\cline { 2 - 4 } KDE $(\%)$ & $<0.213$ & $>0.213$ & $>0.500$ \\
\hline 10 & 1.0 & 11.6 & 9.1 \\
20 & 3.1 & 26.6 & 20.3 \\
30 & 5.7 & 44.1 & 34.9 \\
40 & 8.3 & 64.2 & 52.2 \\
50 & 12.3 & 86.4 & 69 \\
60 & 19 & 11.4 & 84.3 \\
70 & 28.8 & 140.8 & 101 \\
80 & 42.5 & 178.9 & 123.3 \\
90 & 64.4 & 236.9 & 152.4 \\
95 & 80.9 & 294.7 & 178 \\
\hline
\end{tabular}

Note: 0.213 is the minimum training presence suitability threshold. 
give valuable results with patch-abundance data, such as in ponds surrounded by terrestrial lands in which organisms have secretive habits. This approach offers a valuable complement or alternative to other quantitative methodologies, such as landscape modelling and genetics (Manel and Holderegger 2013), particularly when focusing on the most relevant populations and maintaining connectivity between sites is targeted as a priority.

\section{Kernel density estimators and conservation}

Determining the abundance of pond-breeding amphibians over wide ranges, i.e., at the landscape scale, is rarely feasible through methods other than population estimations during their breeding period (Denoël and Lehmann 2006). Indeed, pond-breeding amphibians have more secretive habits during their terrestrial than aquatic stage and the use of radio-telemetry methods can only be applied to a handful of focal populations (Schabetsberger et al. 2004). Using KDEs on patchdistributed estimates of abundances, it was possible to delineate sets of surface areas that differ in their overall probability of encompassing determined fractions of the whole number of reproductive individuals present in the study area. In this perspective, the use of KDEs made it possible to objectively identify the areas needed to maintain a required proportion of individuals. Systematic conservation planning requires the identification of explicit and quantifiable conservation goals (Margules and Pressey 2000), and KDEs provide figures that can be easily incorporated into conservation planning.

KDE results can provide an explicit assessment of quantitative conservation targets, but it is important to integrate them with other conservation approaches, such as the distribution of suitable environments, and other economic and social factors (Margules and Pressey 2000, Compton et al. 2007). However, in the present case, the entire area is encompassed in traditionally managed landscapes, i.e., habitats that are favorable for amphibians and most likely do not constitute barriers to their movements (Hartel et al. 2010). Ecological niche models confirmed that landscape had only a weak effect on the distribution of the paedomorphs in Larzac (Appendix). Actually, $70 \%$ of the study area apparently showed suitability for paedomorphic L. helveticus (Fig. 2C). As the complete protection of the whole area is probably not feasible, KDEs represent a valuable method for delineating protected areas with a primary objective to maintain clusters of targeted sites. Considering landscape resistance and barriers certainly increases the correspondence between KDEs and the actual landscape (Compton et al. 2007, Benhamou and Cornélis 2010). Analyses excluding and including major barriers gave similar results, but choosing the KDEs with barriers can help focus on the most valuable areas. Indeed, KDEs without barriers encompass areas outside barriers, such as inside canyons in the studied case. No newts were found in these areas, which can be considered unsuitable for them. Comparing both approaches allows evaluation of alternative conservation actions depending on possibilities. Nevertheless, the results of the two analyses are mostly congruent, suggesting that this approach is robust.

Integrating KDE with the output of niche models showed that not all areas within kernels have the same suitability for paedomorphs. This integrated approach gave consistent results with the analysis integrating barriers in KDE: The limits of plateaus had a major effect on the distribution of newts. The integrated map (Fig. 2D) more accurately delineated the limits of the plateau, if compared with the analysis of barriers. KDE with barriers had constraints to segment length because each segment of barriers must be more than three times the bandwidth $h$ (Benhamou and Cornélis 2010). In our study system, such segments were at least $4.8 \mathrm{~km}$ long thus hampering to closely follow the topographic barriers. Additional topographical features were highlighted inside the kernels such as some high slopes. Our model was built on the basis of the presence of paedomorphs in populations, therefore steep areas, where pond presence is unlikely, are identified as unsuitable. Nevertheless, newts may use them during their terrestrial stage (Vilter and Vilter 1962). Amphibian populations often exist within networks of populations linked by dispersal (e.g., metapopulations), therefore areas apparently unsuitable for breeding may also be important corridors, needed for long time persistence (Marsh and Trenham 2001). These apparently unsuitable areas within kernels may therefore act as corridors linking the major core areas. In other words, ecological niche models could help at defining target conservation areas to maintain suitable landscapes for newt persistence, whereas kernels help at focusing on the relevant areas to protect and in maintaining connectivity between sites. These two approaches are thus complementary for a more efficient conservation management.

The best conservation performance was obtained by integrating KDE with landscape information, either through the explicit definition of ecological barriers, either through the use of ENM (Fig. 4). Overall, the two approaches provide similar results for practical planning (see Fig. 2), and may be appropriate under different conditions. On the one hand, KDE with barriers may be particularly useful if there are major topographic and anthropic barriers (e.g., roads), that can be objectively identified. If such objective delineation is not feasible, the integration between KDE and ENM would provide an excellent alternative, because it jointly considers the dispersal and the habitat issues. Our approach for the selection of the optimal conservation areas has some limitations. First, we estimated performance on the basis of the ratio between the proportion of protected paedomorphs and the amount of study area requiring protection. In principle, such performance values might be maximized by protecting very small areas, with values 
insufficient to protect viable populations. It is therefore extremely important to combine performance metrics with additional parameters, such as biological information on the surface area required to maintain populations in the long term. Second, the results of KDE analyses may be affected by the choice of bandwidth. Nevertheless, the bandwidth value selected by the ad hoc method corresponded well to the known dispersal range of the studied species (Denoël and Lehmann 2006), suggesting that the used bandwidth value was not unlinked to species biological requirements. Third, performance analysis was based on cross-validation, as no independent data were available, and this might slightly inflate the performance measures. However, the results of performance analysis are likely reliable, because our sampling was nearly exhaustive, therefore it is unlikely that our metrics are biased by missing multiple major areas with paedomorphs. Furthermore, tests with truly independent data sets confirmed that cross-validation approaches provide good measures of relative performance (Barbet-Massin and Jetz 2014).

\section{Conservation areas for rare phenotypes}

Previous studies have highlighted that polyphenisms are geographically clustered (Denoël 2007, Emel and Bonett 2011). Using KDEs in the present study made it possible to quantitatively delineate distribution areas with the highest numbers of paedomorphs in Larzac. This clustering may arise from two non-exclusive processes. First, environmental features favorable to the expression of paedomorphosis may be clustered. Furthermore, given that paedomorphosis has been shown to have a genetic basis (Johnson and Voss 2013), it can also be expected that close populations share some genetic underpinning and thus that the maintenance of paedomorphosis can be favored through gene flow between sites. Now that molecular markers such as microsatellites are available for palmate newts, the use of landscape genetics within a spatial context would be promising to explain the current spatial pattern highlighted by KDE (Drechsler et al. 2013).

Long-term studies showed that extirpation of paedomorphic palmate newts occurred in Larzac (Denoël et al. 2005). Consequently, the resilience of paedomorphosis might only be possible if emigration is maintained from the surrounding environment. Maintaining a high number of ponds is thus particularly important, provided that the connectivity among sites is ensured. Using the areas identified through $95 \% \mathrm{KDE}$, it is possible to maintain the connection among all the main ponds, whereas using $80 \% \mathrm{KDE}$ creates splits among areas. These guidelines correspond at protecting areas covering $95 \%$ and $80 \%$ of the populations of paedomorphs, respectively. Yet, each disjoint area maintains multiple breeding patches. The non-linear increase of the average number of ponds by disjoint area indicates that some thresholds should be considered in establishing the limits of conservation areas. For instance, at $50 \% \mathrm{KDE}$, only five ponds per disjoint area are included, whereas if the $80 \%$ delineation is followed, around 15 ponds would be protected, doubling the surface area required. Off course, the number of disjoint areas is only one of the parameters that should be considered. Actually, a small number of disjoint areas may be achieved by protecting a very small portion of the landscape (Fig. 3D), which would not ensure a sufficient protection to the target species. It is therefore essential to focus first on the number of protected ponds and then on the number of disjoint areas. KDEs allows thus to pinpoint the most valuable areas, i.e., those covering the highest number of ponds with paedomorphs within the smallest surface (Fig. 2).

The areas delineated by KDEs include also many ponds in which no paedomorphs were found $(78 \%$ of the 277 studied ponds were devoid paedomorphs). The absence of paedomorphic individuals in these habitats is caused by a variety of factors (Denoël and Ficetola 2014). For instance, shallow ponds could dry up and thus host metamorphic individuals only. Managements of these ponds within the targeted KDEs could also help to provide adequate habitats in which paedomorphosis could be expressed.

\section{Conclusions}

Delineating conservation areas is a major challenge to sustaining biodiversity. Depending on priorities as well as financial and practical possibilities, this can be achieved using a variety of methods (O'Brien et al. 2012, Gleason et al. 2013). Methods mapping density probabilities across space, such as KDEs based on patch-occupancy data, can provide the grounds for delineations that encompass the richer areas while optimizing connections between the largest groups. Only recently have KDEs been used on the basis of population density and not individual patterns (O'Brien et al. 2012, Martins et al. 2013). The use of KDEs in these studies, along with the one used in the present study for a patch-breeding phenotype, shows their relevance in this context. The development of varied statistical methods in statistical packages such as the open source R software (Calenge 2011), including improvements such as the inclusion of barriers (Compton et al. 2007, Benhamou and Cornélis 2010), and the integration with the more widely used ecological niche models, now makes KDEs useful tools in addressing these questions in a wide variety of organisms.

\section{ACKNOWLEDGMENTS}

We are grateful to C. Bary, M. Bichot, C. Houdoin, A. Lagaly, and L. Winandy for field help; two anonymous reviewers for their constructive comments on the manuscript; A. Alves, B. Azema, J. and C. Bara, J. M. Buresi, D. Bazin de Caix, V. Bourrier, A. Caisso, B. Clarissac, D. Desfères, J. Durand, L. Fabre, J. P. Fabrègues, P. O. Gaignart, M. Geneiz, J. C. Gobart, C. Magne, A. Milhaud, C. Petit, B. Pradel, L. Serieys, S. and O. Sennet, J. L. Soulié, municipalities and Office National des Forêts for allowing access to their ponds. Capture permits covered all the study area and research period (DREAL 
Languedoc-Roussillon). All manipulations followed ethical standards. This is a publication of the Applied and Fundamental Fish Research Center (AFFISH-RC). This research benefited from a F.R.S.-FNRS (Fonds de la Recherche scientifique) grant J.0008.13 and a Fonds Spéciaux pour la Recherche grant $\mathrm{C} 11 / 23$ (University of Liège). M. Denoël is a Senior Research Associate at the F.R.S.-FNRS. The authors declare they have no conflict of interest.

\section{Literature Cited}

Araujo, M. B., and P. H. Williams. 2000. Selecting areas for species persistence using occurrence data. Biological Conservation 96:331-345.

Arntzen, J. W. 2002. Testing for equal catchability of Triturus newts by dip netting. Journal of Herpetology 36:272-276.

Barbet-Massin, M., and W. Jetz. 2014. A 40-year, continentwide, multispecies assessment of relevant climate predictors for species distribution modelling. Diversity and Distributions 20:1285-1295.

Barry, R. P., and J. McIntyre. 2011. Estimating animal densities and home range in regions with irregular boundaries and holes: a lattice-based alternative to the kernel density estimator. Ecological Modelling 222:1666-1672.

Benhamou, S., and D. Cornélis. 2010. Incorporating movement behavior and barriers to improve kernel home range space use estimates. Journal of Wildlife Management 74:13531360.

Calenge, C. 2011. Home range estimation in R: the adehabitatHR package. http://cran.r-project.org/web/packages/ adehabitatHR/vignettes/adehabitatHR.pdf

Compton, B. W., K. McGarigal, S. A. Cushman, and L. R. Gamble. 2007. A resistant-kernel model of connectivity for amphibians that breed in vernal ponds. Conservation Biology 21:788-789.

Crandall, K. A., O. R. P. Bininda-Emonds, G. M. Mace, and R. K. Wayne. 2000. Considering evolutionary processes in conservation biology. Trends in Ecology \& Evolution 15:290-295.

Cushman, S. A., E. L. Landguth, and C. H. Flather. 2012. Evaluating the sufficiency of protected lands for maintaining wildlife population connectivity in the U.S. northern Rocky Mountains. Diversity and Distributions 18:873-884.

Denoël, M. 2007. Priority areas of intraspecific diversity: Larzac, a global hotspot for facultative paedomorphosis in amphibians. Animal Conservation 10:110-118.

Denoël, M., G. Džukić, and M. L. Kalezić. 2005. Effect of widespread fish introductions on paedomorphic newts in Europe. Conservation Biology 19:162-170.

Denoël, M., and G. F. Ficetola. 2014. Heterochrony in a complex world: disentangling environmental processes of facultative paedomorphosis in an amphibian. Journal of Animal Ecology 83:606-615.

Denoël, M., G. F. Ficetola, R. Ćirović, D. Radović, G. Džukić, M. L. Kalezić, and T. D. Vukov. 2009. A multi-scale approach to facultative padomorphosis of European newts in the Montenegrin karst: distribution pattern, environmental variables and conservation. Biological Conservation 142:509-517.

Denoël, M., and A. Lehmann. 2006. Multi-scale effect of landscape processes and habitat quality on newt abundance: implications for conservation. Biological Conservation 130:495-504.

Drechsler, A., D. Geller, K. Freund, S. Künzel, O. Rupp, D. Schmeller, M. Denoël, S. Carranza, and S. Steinfartz. 2013. What remains of a 454 run: a microsatellite loci perspective on three selected amphibian newt species (Calotriton asper, Lissotriton helveticus, Triturus cristatus). Ecology and Evolution 3:3947-3957.

Elith, J., et al. 2006. Novel methods improve prediction of species' distributions from occurrence data. Ecography 29:129-151.
Elith, J., S. J. Phillips, T. Hastie, M. Dudik, Y. En Chee, and C. J. Yates. 2011. A statistical explanation of MaxEnt for ecologists. Diversity and Distributions 17:43-57.

Emel, S. L., and R. M. Bonett. 2011. Considering alternative life history modes and genetic divergence in conservation: a case study of the Oklahoma salamander. Conservation Genetics 12:1243-1259.

Ficetola, G. F., A. Bonardi, C. A. Mucher, N. L. M. Gilissen, and E. Padao-Schipoa. 2014. How many predictors in species distribution models at the landscape scale? Land use versus LiDAR-derived canopy height. International Journal of Geographical Information Science 28:1723-1739.

Gabrion, J., P. Sentein, and C. Gabrion. 1977. Les populations néoténiques de Triturus helveticus Raz. des Causses et du BasLanguedoc. I. Répartition et caractéristiques. Terre et Vie 31:489-506.

Geniez, P., and M. Cheylan. 2012. Les amphibiens et les reptiles $\mathrm{du}$ Languedoc-Roussillon et régions limitrophes. Atlas biogéographique. Biotope \& Museum National d'Histoire Naturelle, Paris, France.

Gleason, M., et al. 2013. Designing a network of marine protected areas in California: Achievements, costs, lessons learned, and challenges ahead. Ocean and Coastal Management 74:90-101.

Hart, K. M., D. G. Zawada, I. Fujisaki, and B. H. Lidz. 2013. Habitat use of breeding green turtles Chelonia mydas tagged in Dry Tortugas National Park: making use of local and regional MPAs. Biological Conservation 161:142-154.

Hartel, T., O. Schweiger, K. Öllerer, D. Cogalniceanu, and J. W. Arntzen. 2010. Amphibian distribution in a traditionally managed rural landscape of Eastern Europe: probing the effect of landscape composition. Biological Conservation 143:1118-1124.

Hijmans, R. J. 2013. Raster: geographic data analysis and modeling. R package version 2.1-49. http://CRAN.R-project. org/package=raster

Hijmans, R. J., S. Pjilips, and J. Elith. 2013. Dismo: species distribution modeling. $\mathrm{R}$ package version 0.9-3. http:// CRAN.R-project.org/package=dismo

Johnson, C. K., and S. R. Voss. 2013. Salamander paedomorphosis. Linking thyroid hormone to life history and life cycle evolution. Pages 229-258 in Y.-B. Shi, editor. Animal metamorphosis. Current topics in developmental biology. Volume 103. Academic Press, Burlington, Massachusetts, USA.

Kanagaraj, R., T. Wiegand, S. Kramer-Schadt, and S. P. Goyal. 2013. Using individual-based movement models to assess inter-patch connectivity for large carnivores in fragmented landscapes. Biological Conservation 167:298309.

Kramer-Schadt, S., et al. 2013. The importance of correcting for sampling bias in MaxEnt species distribution models. Diversity and Distributions 19:1366-1379.

Kremen, C., et al. 2008. Aligning conservation priorities across taxa in Madagascar with high-resolution planning tools. Science 320:222-226.

Manel, S., and R. Holderegger. 2013. Ten years of landscape genetics. Trends in Ecology and Evolution 28:614-621.

Manel, S., H. C. Williams, and S. J. Ormerod. 2001. Evaluating presence-absence models in ecology: the need to account for prevalence. Journal of Applied Ecology 38:291-931.

Margules, C. R., and R. L. Pressey. 2000. Systematic conservation planning. Nature 405:243-253.

Marsh, D. M., and P. C. Trenham. 2001. Metapopulation dynamics and amphibian conservation. Conservation Biology $15: 40-49$.

Martins, C. C. A., A. Andriolo, M. H. Engel, P. G. Kinas, and C. H. Saito. 2013. Identifying priority areas for humpback whale conservation at Eastern Brazilian Coast. Ocean and Coastal Management 75:63-71. 
Mazerolle, M. J., L. L. Bailey, W. L. Kendall, J. A. Royle, S. J. Converse, and J. D. Nichols. 2007. Making great leaps forward: accounting for detectability in herpetological field studies. Journal of Herpetology 41:672-689.

Myers, N., R. A. Mittermeier, C. G. Mittermeier, G. A. B. da Fonseca, and J. Kent. 2000. Biodiversity hotspots for conservation priorities. Nature 403:853-858.

Naidoo, R., A. Balmford, R. Costanza, B. Fisher, R. E. Green, B. Lehner, T. R. Malcolm, and T. H. Ricketts. 2008. Global mapping of ecosystem services and conservation priorities. Proceedings of the National Academy of Sciences USA 105:9495-9500.

Nogués-Bravo, D. 2009. Predicting the past distribution of species climatic niches. Global Ecology and Biogeography 18:521-531.

O'Brien, S. H., A. Webb, M. J. Brewer, and J. B. Reid. 2012. Use of kernel density estimation and maximum curvature to set marine protected area boundaries: identifying a special protection area for wintering red-throated divers in the UK. Biological Conservation 156:15-21.

Pearson, R. G. 2011. Species' distribution modeling for conservation educators and practitioners. Lessons in Conservations 3:54-98.

Pearson, R. G., C. J. Raxworthy, M. Nakamura, and A. T. Peterson. 2007. Predicting species distributions from small numbers of occurrence records: a test case using cryptic geckos in Madagascar. Journal of Biogeography 34:102-117.

Phillips, S. J., R. P. Anderson, and R. E. Schapire. 2006. Maximum entropy modeling of species geographic distributions. Ecological Modelling 190:231-259.

Phillips, S. J., M. Dudik, J. Elith, C. H. Graham, A. Lehmann, J. Leathwick, and S. Ferrier. 2009. Sample selection bias and presence-only distribution models: implications for background and pseudo-absence data. Ecological Applications 19:181-187.

Pinheiro, J., D. Bates, S. DebRoy, and D. Sarkar. 2014. Linear and nonlinear mixed effects models. R package version 3.1. http://cran.r-project.org/web/packages/nlme

$\mathrm{R}$ Core Development Team. 2013. R 3.0.2. R Project for Statistical Computing, Vienna, Austria. www.r-project.org

Ronconi, R. A., B. G. Lascelles, G. M. Langham, J. B. Reid, and D. Oro. 2012. The role of seabirds in Marine Protected Area identification, delineation, and monitoring: introduction and synthesis. Biological Conservation 156:1-4.

Schabetsberger, R., R. Jehle, A. Maletzky, J. Pesta, and M. Sztatecsny. 2004. Delineation of terrestrial reserves for amphibians: post-breeding migrations of Italian crested newts (Triturus c. carnifex) at high altitude. Biological Conservation 117:95-104.

Schofield, G., R. Scott, A. Dimadi, S. Fossette, K. A. Katselidis, D. Koutsoubas, M. K. S. Lilley, J. D. Pantis, A. D. Karagouni, and G. C. Hays. 2013. Evidence-based marine protected area planning for a highly mobile endan- gered marine vertebrate. Biological Conservation 161:101109.

Seaman, D. E., and R. A. Powell. 1996. An evaluation of the accuracy of kernel density estimators for home range analysis. Ecology 77:2075-2085.

Segelbacher, G., S. A. Cushman, B. K. Epperson, M. J. Fortin, O. François, O. J. Hardy, R. Holderegger, P. Taberlet, L. P. Waits, and S. Manel. 2010. Applications of landscape genetics in conservation biology: concepts and challenge. Conservation Genetics 11:375-385.

Semlitsch, R. D. 1987. Paedomorphosis in Ambystoma talpoideum: effects of density, food, and pond drying. Ecology 68:994-1002.

Sillero, N., et al. 2014. Updated distribution and biogeography of amphibians and reptiles of Europe. Amphibia-Reptilia 35:1-31.

Smith, M. A., and D. M. Green. 2005. Dispersal and the metapopulation paradigm in amphibian ecology and conservation: are all amphibian populations metapopulations? Ecography 28:110-128.

Tarjuelo, I., D. Posada, K. A. Crandall, M. Pascual, and X. Turon. 2004. Phylogeography and speciation of colour morphs in the colonial ascidian Pseudotistoma crucigaster. Molecular Ecology 13:3125-3136.

Vilter, A., and V. Vilter. 1962. Migration de reproduction chez le triton alpestre des Alpes vaudoises. Comptes Rendus de l'Academie des Sciences 156:2005-2007.

Wake, D. B., and V. T. Vredenburg. 2008. Are we in the midst of the sixth mass extinction? A view from the world of amphibians. Proceedings of the National Academy of Sciences USA 105:11466-11473.

Warren, D. L. 2012. In defense of "niche modeling". Trends in Ecology \& Evolution 27:497-500.

Warren, D. L., and S. N. Seifert. 2011. Ecological niche modeling in Maxent: the importance of model complexity and the performance of model selection criteria. Ecological Applications 21:335-342.

Wells, K. D. 2007. The ecology and behavior of amphibians. University of Chicago Press, Chicago, Illinois, USA.

Whiteman, H. H. 1994. Evolution of facultative paedomorphosis in salamanders. Quarterly Review of Biology 69:205221.

Wilbur, H. M. 1997. Experimental ecology of food webs: complex systems in temporary ponds. Ecology 78:2279-2302.

Wilson, L. J., C. A. McSorley, C. M. Gray, B. J. Dean, T. E. Dunn, A. Webb, and J. B. Reid. 2009. Radio-telemetry as a tool to define protected areas for seabirds in the marine environment. Biological Conservation 142:1808-1817.

Worton, B. J. 1989. Kernel methods for estimating the utilization distribution in home-range studies. Ecology 70:164-168.

Worton, B. J. 1995. Using Monte Carlo simulation to evaluate kernel-based home range estimators. Journal of Wildlife Management 59:794-800.

\section{Supplemental Material}

Ecological Archives

The Appendix is available online: http://dx.doi.org/10.1890/14-1041.1.sm 